\title{
Diabetes Mellitus, Insulin, Sulfonylurea and Advanced Fibrosis in Non- Alcoholic Fatty Liver Disease
}

George Boon-Bee Goh'1, Mangesh R Pagadala', Jaividhya Dasarathy ${ }^{3}$, Aynur Unalp-Arida ${ }^{7}$, Ruth Sargent ${ }^{1}$, Carol Hawkins ${ }^{2}$, Achuthan Sourianarayanane ${ }^{1}$, Amer Khiyami ${ }^{5}$, Lisa Yerian ${ }^{4}$, Rish K Pai ${ }^{4}$, Srinivasan Dasarathy ${ }^{1,6}$ and Arthur $\mathrm{J} \mathrm{McCullough}^{1,6 *}$

${ }^{1}$ Departments of Gastroenterology at Cleveland Clinic, Johns Hopkins University, USA

${ }^{2}$ MetroHealth Medical Center, Johns Hopkins University, USA

${ }^{3}$ Department of Family Medicine at MetroHealth Medical Center, Johns Hopkins University, USA

${ }^{4}$ Departments of Pathology at Cleveland Clinic, Johns Hopkins University, USA

${ }^{5}$ MetroHealth Medical Center, Johns Hopkins University, USA

${ }^{6}$ Department of Pathobiology at Cleveland Clinic, Johns Hopkins University, USA

${ }^{7}$ Department of Public Health, Johns Hopkins University, USA

\begin{abstract}
Background \& aims: Diabetes mellitus is a risk factor for advanced fibrosis in non-alcoholic fatty liver disease. However, not all non-alcoholic fatty liver disease patients with diabetes develop advanced fibrosis. We hypothesised that prescription medications used by these patients influence the development of advanced fibrosis. We investigated the association of commonly used medications and advanced fibrosis in non-alcoholic fatty liver disease patients with diabetes.
\end{abstract}

Methods: Clinical information including demographics, medical history, medication history, biochemical and histologic variables were ascertained in 459 patients with biopsy proven non-alcoholic fatty liver disease. We compared characteristics of patients with and without diabetes and explored potential associations between classes of drugs as risk factors and advanced fibrosis among the diabetic patients with NAFLD.

Results: Presence of diabetes was an independent risk factor for advanced fibrosis. In diabetic patients, age (OR 1.09; 95\% Cl 1.04-1.15, $\mathrm{p}=0.000$ ) and grade of ballooning (OR 5.59;95\% $\mathrm{Cl} 2.69-11.61, \mathrm{p}=0.000$ ) had a positive relationship with advanced fibrosis. The use of insulin (OR 4.95; $95 \% \mathrm{Cl} 1.65-14.88, p=0.004)$ and sulfonylurea (OR $5.07 ; 95 \% \mathrm{Cl} 1.87-13.75, \mathrm{p}=0.001)$ were positively associated while statin use (OR $0.31 ; 95 \% \mathrm{Cl} 0.12-0.78, \mathrm{p}=0.013)$ was negatively associated with advanced fibrosis.

Conclusion: Among non-alcoholic fatty liver disease patients with diabetes, the prevalence of advanced fibrosis was higher in patients treated with insulin and sulfonylurea, but lower in patients on statins. These findings provide support for a greater role of statin use in non-alcoholic fatty liver disease patients with diabetes while limiting the use of insulin and sulfonylurea.

Keywords: NAFLD; Fibrosis; Diabetes; Insulin; Sulfonylureas; Statins

\begin{abstract}
Abbreviations: NAFLD: Non-Alcoholic Fatty Liver Disease; NASH: Non-Alcoholic SteatoHepatitis; ACE-I: type 2 diabetes mellitus (DM), Angiotensinogen Converting Enzyme Inhibitor; ADA: American Diabetes Association; JNC: Joint National Committee; BMI: Body Mass Index; ALP: Alkaline phosphatase; ALT: Alanine aminotransferase; AST: Aspartate aminotransferase; INR: International Normalised Ratio; Chol: Total Cholesterol; TG: Triglycerides; HDL: High Density Lipoprotein Cholesterol; LDL: Low Density Lipoprotein Cholesterol; ARB: Angiotensin II receptor blocker; SD: Standard deviations; ORs: Odds ratios; CI: Confidence Intervals; NAS: NAFLD activity score; IGF-1: Insulin-like growth factor; HSC: Hepatic Stellate Cells; PI3K: Phosphatidyl inositol 3 kinase; ERK: Extracellular sSignal Related Kinase; CTGF: Connective Tissue Growth Factor; HCC: HepatoCellular Carcinoma
\end{abstract}

\section{Introduction}

Non-Alcoholic Fatty Liver Disease (NAFLD) represents a spectrum of histologic abnormalities ranging from steatosis alone to NonAlcoholic Steatohepatitis (NASH), to advanced fibrosis and cirrhosis [1]. Patients with type 2 Diabetes Mellitus (DM) have an increased the risk of developing NAFLD while significantly increasing the risk of NASH and hepatic fibrosis/cirrhosis [2,3]. NAFLD patients with DM have twice the mortality compared to non-diabetic NAFLD patients [4].
Multiple studies have examined anti-diabetic, lipid lowering and antihypertensive medications for treating NAFLD, but these studies did not identify a clear or consistent benefit on hepatic fibrosis [5]. However, not all subjects with diabetes develop advanced fibrosis or cirrhosis. We hypothesised that certain medications used by these patients may have influenced the development of advanced fibrosis in NAFLD patients with DM, as has been observed in other chronic liver disease. The use of Angiotensinogen Converting Enzyme Inhibitor (ACE-I) was observed to reduce the risk of advanced fibrosis in chronic hepatitis $\mathrm{C}$ patients [6]. Similarly, the use of metformin was associated with a decrease in risk of hepatocellular carcinoma while insulin/sulfonylurea therapy was reported to increase this risk [7]. The present study sought to evaluate

*Corresponding author: Arthur J McCullough, Department of Gastroenterology, Cleveland Clinic Foundation 9500 Euclid Avenue/ A30, Cleveland, OH 44195, USA Tel: 1-216-444-6521; Fax: 1-216-444-3889; E-mail: mcculla@ccf.org

Received June 27, 2014; Accepted July 26, 2014; Published August 03, 2014

Citation: Goh GBB, Pagadala MR, Dasarathy J, Unalp-Arida A, Sargent $R$ et al. (2014) Diabetes Mellitus, Insulin, Sulfonylurea and Advanced Fibrosis in Non-Alcoholic Fatty Liver Disease. J Diabetes Metab 5: 410 doi:10.4172/21556156.1000410

Copyright: $\odot 2014$ Goh GBB, et al. This is an open-access article distributed under the terms of the Creative Commons Attribution License, which permits unrestricted use, distribution, and reproduction in any medium, provided the original author and source are credited. 
the relationship between DM and advanced fibrosis in NAFLD patients and explore the association of commonly used medications and advanced fibrosis in NAFLD

\section{Methods}

\section{Study population}

The study ascertained data from patients aged 18 years and over with histologically proven NAFLD from two hepatology clinics in Cleveland, Ohio (Cleveland Clinic Foundation and MetroHealth Medical Centre). Patients with prior therapies that may be beneficial for NAFLD such as vitamin E, pentoxifylline, pioglitazone, prescribed diet and exercise weight loss programs were excluded. Patients with other potential contributory causes of liver disease (alcohol consumption [ $>21$ drinks and $>14$ drinks per week for males and females respectively], hepatotoxic drug history, chronic viral hepatitis, hemochromatosis, autoimmune hepatitis, Wilson's disease or alpha-1 antitrypsin disease) were excluded [8].

\section{Study design}

Demographic and clinical information was gathered from an electronic medical record system that is common to both hospitals. Diabetes mellitus was diagnosed by American Diabetes Association (ADA) criteria with or without the use of antidiabetic medications [9]. Hypertension was diagnosed by the Joint National Committee (JNC) 7 criteria [10]. All diagnoses were also verified based on documentation in the electronic medical records by one of the investigators (AJM or $\mathrm{SD}$ ). Anthropometric measurements including height, weight and Body Mass Index (BMI) were recorded. Laboratory data including liver function tests [serum albumin, bilirubin, Alkaline Phosphatase (ALP), Alanine Aminotransferase (ALT), Aspartate Aminotransferase (AST)], platelet count, International Normalised Ratio (INR) and markers of the metabolic syndrome (total Cholesterol (Chol), Triglycerides (TG), High Density Lipoprotein (HDL) cholesterol, Low Density Lipoprotein (LDL) cholesterol, HbA1C) were collated. Liver biopsies were evaluated using the Non-alcoholic Steatohepatitis Clinical Research Network criteria [11]. In brief, the biopsy was scored for steatosis, lobular inflammation, ballooning and fibrosis. Stage 3 (bridging fibrosis) and 4 (cirrhosis) were classified as advanced fibrosis. Only clinical data obtained within 6 months of the corresponding liver biopsy were included. A careful medication history focused on the use of insulin, metformin, sulfonylurea, hydroxyl-methylglutaryl-coenzyme A reductase inhibitors (statins) and angiotensin converting enzyme inhibitor (ACE-I)/Angiotensin II Receptor Blocker (ARB) because these agents have been shown to affect insulin resistance, hepatic lipid metabolism or fibrosis. Approval of the study protocol was obtained from the Institutional Review Board in accordance with the ethical guidelines of the declaration of Helsinki.

\section{Statistical analysis}

Descriptive statistics were computed for all variables; means with Standard Deviations (SD) and frequencies with percentages for continuous and categorical variables respectively. Differences between patients with and without DM were analysed using the Students T test and the Pearson's Chi-square test for continuous and categorical variables respectively. In addition, similar analysis for differences between patients with and without advanced fibrosis (fibrosis stage 0-2 vs. stage 3-4) in diabetic subjects was also performed. Independent factors associated with advanced fibrosis were assessed using binary logistic regression multivariate analysis in both total cohort and in the subgroup of DM patients. Factors thought to be associated with advanced fibrosis in NAFLD including age, BMI, gender, presence of hypertension/DM, lipid profile and liver histology (grade of steatosis, inflammation and ballooning) were included in the model. The magnitude of the associations was assessed by Odds Ratios (ORs) and corresponding 95\% Confidence Intervals (CI). This multivariate analysis of the cohort was internally validated using the cross-validation technique (80:20 cross validation). SPSS version 21 (Chicago, Illinois, USA) statistical software package was used to conduct the statistical computing. All $\mathrm{P}$ values quoted were two-sided with $\mathrm{P}<0.05$ considered statistically significant.

\section{Results}

Available data from 459 patients with biopsy proven NAFLD were reported. As shown in Table 1, the mean age was $49 \pm 12$ years and $60.7 \%$ were female. The majority of patients were obese with hypertension and DM present in $56.4 \%$ and $47.9 \%$ respectively. Advanced fibrosis was present in 132 patients (28.8\%). Abnormal AST and ALT were present in 54 and $58 \%$ of the patients, respectively. A relatively large proportion of the cohort had abnormal TG, HDL cholesterol, and HbA1C values.

\begin{tabular}{|c|c|c|c|c|}
\hline Variable & $\begin{array}{c}\text { Total cohort; } \\
n=459\end{array}$ & $\begin{array}{c}\text { DM patients; } \\
n=220\end{array}$ & $\begin{array}{c}\text { Non-DM patients; } \\
n=239\end{array}$ & $\begin{array}{c}P \\
\text { value }\end{array}$ \\
\hline Age (years) & $49 \pm 12$ & $52 \pm 11$ & $46 \pm 12$ & 0.00 \\
\hline Proportion Female & $278(60.7 \%)$ & $152(69.1 \%)$ & $126(52.9 \%)$ & 0.00 \\
\hline $\mathrm{BMI}\left(\mathrm{kg} / \mathrm{m}^{2}\right)$ & $35.9 \pm 8.5$ & $37.0 \pm 7.9$ & $35.0 \pm 9.0$ & 0.01 \\
\hline Presence of HTN & $259(56.4 \%)$ & $156(70.9 \%)$ & $103(43.1 \%)$ & 0.00 \\
\hline Presence of DM & $220(47.9 \%)$ & NA & NA & - \\
\hline Use of Insulin & $52(11.3 \%)$ & $52(23.7 \%)$ & NA & - \\
\hline Use of Metformin & $94(20.5 \%)$ & $94(42.7 \%)$ & NA & - \\
\hline Use of Sulfonylurea & $67(14.6 \%)$ & $67(30.6 \%)$ & NA & - \\
\hline Use of Statin & $123(26.9 \%)$ & $88(40.2 \%)$ & $35(14.6 \%)$ & 0.00 \\
\hline Use of ACE-I/ARB & $175(38.1 \%)$ & $107(48.6 \%)$ & $68(28.5 \%)$ & 0.00 \\
\hline Serum Bilirubin (mg/dL) & $0.68 \pm 0.40$ & $0.69 \pm 0.43$ & $0.67 \pm 0.38$ & 0.61 \\
\hline AST (U/L) & $56.8 \pm 65.6$ & $54.3 \pm 35.9$ & $59.1 \pm 84.1$ & 0.44 \\
\hline ALT (U/L) & $72.4 \pm 56.4$ & $65.3 \pm 50.1$ & $78.9 \pm 60.9$ & 0.01 \\
\hline Albumin $(\mathrm{g} / \mathrm{dL})$ & $4.2 \pm 0.5$ & $4.1 \pm 0.5$ & $4.3 \pm 0.4$ & 0.01 \\
\hline INR & $1.03 \pm 0.17$ & $1.04 \pm 0.18$ & $1.02 \pm 0.16$ & 0.36 \\
\hline Platelet count (k/uL) & $238.0 \pm 77.3$ & $231.6 \pm 79.1$ & $243.7 \pm 75.3$ & 0.10 \\
\hline Creatinine $(\mathrm{mg} / \mathrm{dL})$ & $0.89 \pm 0.38$ & $0.85 \pm 0.51$ & $0.92 \pm 0.19$ & 0.08 \\
\hline Total cholesterol $(\mathrm{mg} / \mathrm{dL})$ & $198.5 \pm 51.7$ & $193.9 \pm 54.9$ & $202.5 \pm 48.5$ & 0.09 \\
\hline TG (mg/dL) & $207.1 \pm 186.5$ & $232.7 \pm 231.0$ & $184.5 \pm 132.4$ & 0.01 \\
\hline $\mathrm{HDL}(\mathrm{mg} / \mathrm{dL})$ & $42.6 \pm 10.4$ & $41.5 \pm 10.3$ & $43.6 \pm 10.0$ & 0.05 \\
\hline LDL (mg/dL) & $123.6 \pm 43.2$ & $116.4 \pm 45.2$ & $130.0 \pm 40.3$ & 0.00 \\
\hline $\mathrm{HbA} 1 \mathrm{C} \%$ & $6.5 \pm 1.4$ & $7.40 \pm 1.51$ & $5.60 \pm 0.57$ & 0.00 \\
\hline Ferritin (ng/mL) & $231.2 \pm 233.6$ & $206.4 \pm 233.9$ & $253.9 \pm 231.6$ & 0.05 \\
\hline Grade of steatosis & $1.9 \pm 0.8$ & $1.9 \pm 0.8$ & $1.9 \pm 0.8$ & 0.96 \\
\hline $\begin{array}{l}\text { Grade of lobular } \\
\text { inflammation }\end{array}$ & $1.4 \pm 0.7$ & $1.5 \pm 0.6$ & $1.4 \pm 0.7$ & 0.02 \\
\hline Grade of ballooning & $1.1 \pm 0.7$ & $1.2 \pm 0.7$ & $0.9 \pm 0.7$ & 0.00 \\
\hline NAS & $4.5 \pm 1.6$ & $4.7 \pm 1.6$ & $4.3 \pm 1.6$ & 0.01 \\
\hline Stage of fibrosis & $1.6 \pm 1.3$ & $2.0 \pm 1.3$ & $1.1 \pm 1.2$ & 0.00 \\
\hline $\begin{array}{l}\text { Presence of advanced } \\
\text { fibrosis }\end{array}$ & $132(28.8 \%)$ & $91(41.6 \%)$ & $41(17.2 \%)$ & 0.00 \\
\hline
\end{tabular}

Data expressed as mean \pm SD or number and percentages (\%).

SD: Standard Deviation; BMI: Body Mass Index; HTN: Hypertension; DM: Diabetes ACE-I: Angiotensin Converting Enzyme Inhibitor; ARB: Angiotensin II Receptor Blocker; AST: Aspartate aminotransferase; ALT: Alanine aminotransferase; INR International Normalised Ratio; TG: Triglyceride; HDL: High Density Lipoprotein Cholesterol; LDL: Low Density Lipoprotein Cholesterol; NAS: NAFLD activity score; NA: Not applicable

Table 1: Characteristics of NAFLD patients with and without DM. 
Citation: Goh GBB, Pagadala MR, Dasarathy J, Unalp-Arida A, Sargent R, et al. (2014) Diabetes Mellitus, Insulin, Sulfonylurea and Advanced Fibrosis in Non-Alcoholic Fatty Liver Disease. J Diabetes Metab 5: 410 doi:10.4172/2155-6156.1000410

Differences in patients with and without DM are shown in Table 1. Compared to non-DM patients, DM patients were older, a greater proportion were female, had a higher BMI and a higher prevalence of hypertension. They were also more likely to be on a statin or ACE-I/ ARBs. Biochemical parameters including ALT, Albumin, TG, HDL, LDL and $\mathrm{HbA1C}$ were also significantly different in the 2 groups. On liver histology, while there were no differences in steatosis, DM patients had more lobular inflammation and ballooning, as well as a higher NAFLD Activity Score (NAS). Advanced fibrosis was also increased in DM patients.

\begin{tabular}{|l|c|c|c|c|}
\hline \multirow{2}{*}{ Variable } & \multicolumn{3}{|c|}{ Whole cohort } & \multicolumn{2}{c|}{ Cross validation 80:20. } \\
\cline { 2 - 5 } & OR; 95\% CI & P value & OR; 95\% CI & P value \\
\hline Age & $1.05 ; 1.02-1.08$ & 0.002 & $1.07 ; 1.03-1.11$ & 0.000 \\
\hline DM & $2.36 ; 1.27-4.38$ & 0.007 & $2.33 ; 1.15-4.73$ & 0.019 \\
\hline Total Cholesterol & $0.97 ; 0.95-0.99$ & 0.011 & $0.97 ; 0.94-0.99$ & 0.003 \\
\hline LDL Cholesterol & $1.02 ; 1.00-1.04$ & 0.034 & $1.03 ; 1.01-1.05$ & 0.011 \\
\hline Ballooning & $5.59 ; 3.35-9.34$ & 0.000 & $7.77 ; 4.14-14.58$ & 0.000 \\
\hline
\end{tabular}

Covariates included were age, BMI, gender, presence of hypertension, presence of DM, lipids and histology.

OR: Odds ratio; $\mathrm{Cl}$ : Confidence interval; DM: Diabetes mellitus.

Table 2: Independent risk factors of advanced fibrosis in whole cohort.

\begin{tabular}{|c|c|c|c|}
\hline Variable & $\begin{array}{c}\text { Presence of } \\
\text { advanced } \\
\text { fibrosis } n=91\end{array}$ & $\begin{array}{l}\text { Absence of advanced } \\
\text { fibrosis } n=128\end{array}$ & $P$ value \\
\hline Age (years) & $55 \pm 11$ & $50 \pm 11$ & 0.00 \\
\hline Proportion Female (\%) & $63(69.2 \%)$ & $88(68.8 \%)$ & 0.94 \\
\hline BMI $\left(\mathrm{kg} / \mathrm{m}^{2}\right)$ & $36.3 \pm 7.8$ & $37.4 \pm 8.05$ & 0.32 \\
\hline Presence of HTN (\%) & $71(78 \%)$ & $84(65.6 \%)$ & 0.05 \\
\hline Duration of DM (years) & $4.9 \pm 6.3$ & $4.6 \pm 5.1$ & 0.77 \\
\hline Use of Insulin (\%) & $26(20.3 \%)$ & $26(28.9 \%)$ & 0.14 \\
\hline Use of Metformin (\%) & $39(42.9 \%)$ & $54(42.2 \%)$ & 0.92 \\
\hline Use of Sulfonylurea (\%) & $35(38.9 \%)$ & $32(25.0 \%)$ & 0.03 \\
\hline Use of Statin (\%) & $31(34.1 \%)$ & $56(44.1 \%)$ & 0.14 \\
\hline Use of Ace-I/ARB (\%) & $41(45.1 \%)$ & $65(50.8 \%)$ & 0.40 \\
\hline Serum Bilirubin (mg/dL) & $0.75 \pm 0.49$ & $0.65 \pm 0.38$ & 0.10 \\
\hline AST (U/L) & $61.6 \pm 37.6$ & $48.9 \pm 33.9$ & 0.01 \\
\hline $\operatorname{ALT}(\mathrm{U} / \mathrm{L})$ & $65.2 \pm 55.9$ & $64.9 \pm 45.7$ & 0.96 \\
\hline Albumin (g/dL) & $4.0 \pm 0.6$ & $4.2 \pm 0.4$ & 0.00 \\
\hline INR & $1.06 \pm 0.14$ & $1.02 \pm 0.20$ & 0.10 \\
\hline Platelet count (k/uL) & $202.0 \pm 87.8$ & $252.0 \pm 65.2$ & 0.00 \\
\hline Creatinine (mg/dL) & $0.84 \pm 0.29$ & $0.87 \pm 0.63$ & 0.62 \\
\hline Total cholesterol (mg/dL) & $178.8 \pm 51.4$ & $203.3 \pm 55.3$ & 0.00 \\
\hline TG (mg/dL) & $204.6 \pm 118.7$ & $251.5 \pm 279.5$ & 0.18 \\
\hline $\mathrm{HDL}(\mathrm{mg} / \mathrm{dL})$ & $39.9 \pm 11.5$ & $42.6 \pm 9.3$ & 0.08 \\
\hline LDL (mg/dL) & $108.0 \pm 41.3$ & $121.3 \pm 47.1$ & 0.06 \\
\hline $\mathrm{HbA1C}(\%)$ & $7.4 \pm 1.4$ & $7.3 \pm 1.5$ & 0.51 \\
\hline Ferritin (ng/mL) & $213.3 \pm 236.3$ & $201.2 \pm 234.2$ & 0.74 \\
\hline Grade of steatosis & $1.9 \pm 0.9$ & $1.9 \pm 0.8$ & 0.85 \\
\hline $\begin{array}{l}\text { Grade of lobular } \\
\text { inflammation }\end{array}$ & $1.5 \pm 0.6$ & $1.5 \pm 0.6$ & 0.66 \\
\hline Grade of ballooning & $1.5 \pm 0.5$ & $1.0 \pm 0.7$ & 0.00 \\
\hline NAS & $5.0 \pm 1.4$ & $4.5 \pm 1.7$ & 0.02 \\
\hline
\end{tabular}

Data expressed as mean \pm SD or number and percentages (\%).

SD: Standard Deviation; BMI: Body Mass Index; HTN: Hypertension; ACE-I: Angiotensin Converting Enzyme Inhibitor; ARB: Angiotensin II Receptor Blocker; AST: Aspartate aminotransferase; ALT: Alanine aminotransferase; INR International Normalised Ratio; TG: Triglyceride, HDL: High Density Lipoprotein Cholesterol; LDL: Low Density Lipoprotein Cholesterol; NAS: NAFLD activity score; NA: Not applicable.

Table 3: Baseline characteristics of DM subjects by presence of advanced fibrosis.

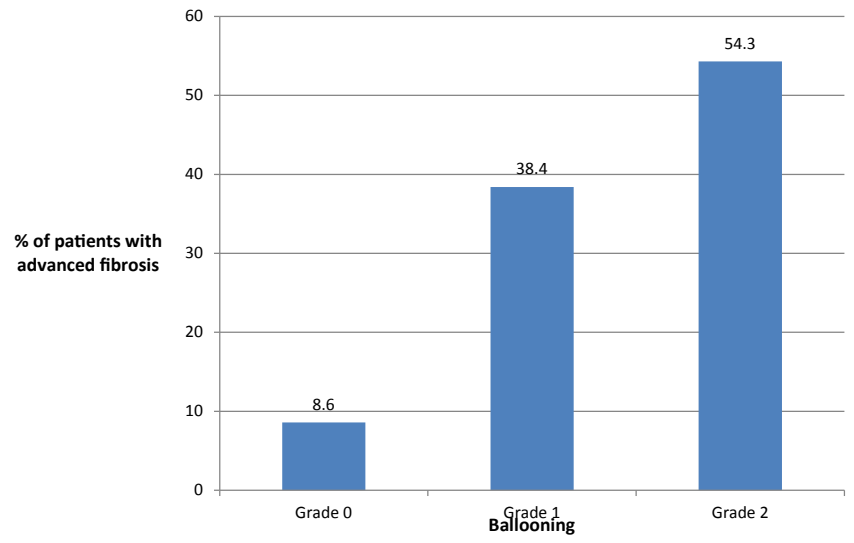

Figure 1: Relationship of grade of ballooning to advanced fibrosis in NAFLD patients with DM.

\begin{tabular}{|l|c|c|c|c|}
\hline \multirow{2}{*}{ Variable } & \multicolumn{2}{|c|}{ DM cohort } & \multicolumn{2}{c|}{ Cross validation 80:20. } \\
\cline { 2 - 5 } & OR; 95\% CI & P value & OR; 95\% CI & P value \\
\hline Age & $1.09 ; 1.04-1.15$ & 0.000 & $1.12 ; 1.06-1.19$ & 0.000 \\
\hline Ballooning & $5.59 ; 2.69-11.61$ & 0.000 & $7.01 ; 3.01-16.33$ & 0.000 \\
\hline Use of Insulin & $4.95 ; 1.65-14.88$ & 0.004 & $4.64 ; 1.35-15.95$ & 0.015 \\
\hline Use of Sulfonylurea & $5.07 ; 1.87-13.75$ & 0.001 & $4.63 ; 1.43-15.00$ & 0.011 \\
\hline Use of Statins & $0.31 ; 0.12-0.78$ & 0.013 & $0.30 ; 0.10-0.86$ & 0.025 \\
\hline
\end{tabular}

Covariates included were age, BMI, gender, presence of hypertension, lipids histology, use of insulin, metformin, sulfonylurea, statin and ACE-I/ARB.

OR: Odds ratio, $\mathrm{Cl}$ : Confidence interval.

Table 4: Independent risk factors of advanced fibrosis in DM subjects.

The independent risk factors for advanced fibrosis in the entire cohort are shown in Table 2 and included the presence of DM, age, ballooning, plasma cholesterol levels which held true during the $80 \%$ : $20 \%$ cross validation.

DM patients with and without advanced fibrosis are compared in Table 3. Compared to patients without advanced fibrosis, patients with advanced fibrosis were older and had a higher incidence of hypertension. There were no significant differences in gender, BMI or duration of DM. On univariate analysis, patients with advanced fibrosis were more likely to be on sulfonylureas while there were no differences in the use of insulin, metformin, statin or ACE-I/ARB. Patients with advanced fibrosis had significantly higher AST levels, while serum albumin and platelet count were significantly lower (Table 3). There were no differences in creatinine, lipids, and $\mathrm{HbAlC}$ or ferritin levels. On liver histology, the grade of ballooning and NAS were significantly higher with advanced fibrosis. Further analysis showed higher grades of ballooning were associated with increasing fibrosis stage as shown in Figure 1 with grades of 0,1 and 2 ballooning associated with a prevalence of advanced cirrhosis of $8.6 \%, 38.4 \%$ and $54.3 \%$, respectively.

Multivariate analysis of advanced fibrosis risk factors in the context of DM are shown in Table 4. Advanced fibrosis was independently affected by age, grade of ballooning, use of insulin, sulfonylurea and statin. Increasing age (OR 1.09; 95\% CI 1.04-1.15, p=0.000) and grade of ballooning (OR 5.59; 95\% CI 2.69-11.61, p=0.000) were positively associated with advanced fibrosis. Use of insulin (OR 4.95; 95\% CI 1.65-14.88, $\mathrm{p}=0.004$ ) and sulfonylurea (OR 5.07; 95\% CI 1.87-13.75, $\mathrm{p}=0.001)$ were also positively associated with advanced fibrosis. In contrast, the use of statins (OR $0.31 ; 95 \%$ CI $0.12-0.78, \mathrm{p}=0.013$ ) was negatively associated with advanced fibrosis. The use of metformin and ACE-I/ARB were not associated with advanced fibrosis. 


\section{Discussion}

In addition to confirming that DM is an independent risk factor for advanced fibrosis in NAFLD, the present study makes the novel observation that advanced fibrosis in NAFLD may be impacted by certain medications used to treat diabetes and dyslipidemia. Among patients with DM, use of insulin and sulfonylurea was positively associated with advanced fibrosis while statin therapy was negatively associated with advanced fibrosis. Age and ballooning were additional independent risk factors for advanced fibrosis.

Risk factors for advanced fibrosis among NAFLD patients identified in the present study are similar to those reported by others [3,12-15]. Even though DM is a recognized risk factor for advanced fibrosis in NAFLD we observed that certain prescribed medications affect the relation between fibrosis and diabetes mellitus [3,12-15].

Major therapeutic approaches in diabetes mellitus include either an increase in circulating insulin levels (exogenous insulin or sulfonylureas) or a decrease in insulin resistance (metformin, thiazolidinediones). Our novel observation that both insulin and sulfonylurea were risk factors for advanced hepatic fibrosis suggests that increasing circulating insulin levels are implicated in the development of fibrosis. Expression of insulin and Insulin-Like Growth Factor (IGF-1) receptors on the collagen producing Hepatic Stellate Cells (HSC) is increased during active fibrogenesis [16]. In addition, insulin and IGF-1 stimulate HSC proliferation in a dose dependent fashion [16]. Furthermore, insulin signalling via the Phosphatidyl Inositol 3 Kinase (PI3K) and extracellular signal related kinase (ERK) increases collagen gene expression [16]. Similarly, glucose and insulin stimulate the expression of connective tissue growth factor [CTGF], which is a peptide growth factor that plays a pivotal role in fibrogenesis [17-19].

Compatible with our findings, is the report that anti-diabetic medications also affect the risk of Hepatocellular Carcinoma [HCC] [7,20-22]. The use of insulin and sulfonylurea was associated with a $161 \%$ and $62 \%$ increased risk of HCC respectively, while the use of metformin was associated with $50 \%$ reduction in the incidence of HCC incidence [23]. While it is commonly postulated that insulin and insulin secretagogues have direct effects of carcinogenesis, it may also be possible that the risk of HCC development is due in part to the insulin/ insulin secretagogues stimulated progression of liver tissue to advanced fibrosis or cirrhosis, especially since HCC typically develops following the sequence of chronic hepatic inflammation to fibrosis and cirrhosis [23,24].

Our study also shows that the use of statins was associated with reduced risk of advanced hepatic fibrosis in NAFLD patients with DM. In the context of NAFLD and hyperinsulinemia, the hepatic accumulation of lipid molecules leads to inflammation and lipotoxicity [25]. Lipotoxicity plays a crucial role in the pathogenesis of hepatic steatosis, steatohepatitis and fibrosis. Similar mechanisms of lipotoxicity injury can also be seen in atherosclerosis and pancreatic $\beta$ cell destruction in DM [25]. Not surprisingly, atherosclerosis and coronary artery disease are closely linked to NAFLD and represent the most frequent cause of death in these patients [26]. On the other hand, statins, being the mainstay of lipid-lowering therapy, may improve liver outcomes by lowering lipids and lipotoxicity [27]. Cholesterol lowering with statins has been reported to reduce cardiovascular risks and mortality in patients with NAFLD [28]. Cholesterol lowering by both statin and statin/ezetimibe combinations has also been shown to improve necroinflammation and reverse hepatic fibrosis in diabetic mice models [29]. However, human studies have not shown clear or consistent beneficial effect of statins in NAFLD [5,27]. In a Swedish study that explored changes in liver histology over time among patients with NAFLD, there was less fibrosis in patients prescribed a statin [30]. Our data in a large cohort of well characterized patients with NAFLD suggests that statin use is accompanied by lower hepatic fibrosis. These data reiterate both the safety and benefit of statins in NAFLD.

This is the first study to demonstrate a direct relation between the use of insulin/sulfonylurea and risk of advanced fibrosis among NAFLD patients with DM. The recommended standard treatment strategy for DM would generally include oral hypoglycaemic agents such as metformin or sulfonylurea initially and only add insulin therapy when oral combination therapy is no longer effective or required with the progressive $\beta$ cell dysfunction seen in later stages of DM [30]. Both sulfonylureas and insulin use have similar associations with advanced fibrosis. This suggests that it is the stimulation of insulin secretion or insulin per se, rather than treatment choices dictated by the severity of DM, that influences advance fibrosis. This is further supported by comparable $\mathrm{HbA} 1 \mathrm{c}$ levels between patients with and without advanced fibrosis. In addition, the duration of DM was not significantly different between patients with and without advanced fibrosis. Furthermore, since medication history reconciliation occurred at every clinic visit and recorded automatically in the electronic medical records of each patient as per standard clinic protocol, records of medication history were robust and accurate. This information was retrieved for each patient to ascertain the use of the various medications of interest.

The potential limitations of our study include the cross sectional nature of the study which allows evaluation of associations only, rather than causal inference. Secondly, the exact duration and dose of drug use were not available. However, the duration of diabetes did not differ between DM patients with and without advanced fibrosis. In addition, all patients were on the minimal effective therapeutic dose and it is difficult to assess the median dose since antidiabetic and antihypertensive medication doses are adjusted during clinical care. Despite these potential limitations, the large number of subjects and careful review of clinical data of patients followed prospectively provides support for our hypothesis that therapies to control diabetes and dyslipidemia may have an effect on hepatic fibrosis in NAFLD. These data lay the foundation for future multicenter network based studies on the impact of medications on liver histology and progression of disease in NAFLD.

\section{Conclusion}

Our study shows that NAFLD patients with DM on insulin and sulfonylurea were more likely to have advanced hepatic fibrosis, while those on statins were less likely to have advanced hepatitis fibrosis. These data have significant clinical implications and provide novel insights into the pathogenesis of fibrosis in these patients. In addition, these findings provide additional support for statin use in NAFLD patients with DM while limiting the use of insulin and sulfonylurea. Prospective studies to evaluate concurrent medication use are warranted to explore this association in greater detail.

\section{Acknowledgements}

GBBG was supported by Singapore SingHealth HMDP. MRP was supported by NIH T32 DK061917. SD, JD, AJM, RS, CH were supported by NIH DK U.

\section{References}

1. Chalasani N, Younossi Z, Lavine JE, Diehl AM, Brunt EM, et al. (2012) The diagnosis and management of non-alcoholic fatty liver disease: practice Guideline by the American Association for the Study of Liver Diseases, American College of Gastroenterology, and the American Gastroenterological Association. Hepatology 55:2005-2023. 
Citation: Goh GBB, Pagadala MR, Dasarathy J, Unalp-Arida A, Sargent R, et al. (2014) Diabetes Mellitus, Insulin, Sulfonylurea and Advanced Fibrosis in Non-Alcoholic Fatty Liver Disease. J Diabetes Metab 5: 410 doi:10.4172/2155-6156.1000410

Page 5 of 5

2. El-Serag HB, Tran T, Everhart JE (2004) Diabetes increases the risk of chronic liver disease and hepatocellular carcinoma. Gastroenterology 126: 460-468.

3. Hossain N, Afendy A, Stepanova M, Nader F, Srishord M, et al. (2009) Independent predictors of fibrosis in patients with nonalcoholic fatty liver disease. Clin Gastroenterol Hepatol 7: 1224-1229, 1229.

4. Younossi ZM, Gramlich T, Matteoni CA, Boparai N, McCullough AJ (2004) Nonalcoholic fatty liver disease in patients with type 2 diabetes. Clin Gastroenterol Hepatol 2: 262-265.

5. Musso G, Gambino R, Cassader M, Pagano G (2010) A meta-analysis of randomized trials for the treatment of nonalcoholic fatty liver disease. Hepatology 52: 79-104

6. Corey KE, Shah N, Misdraji J, Abu Dayyeh BK, Zheng H, et al. (2009) The effect of angiotensin-blocking agents on liver fibrosis in patients with hepatitis C. Liver Int 29: 748-753.

7. Donadon V, Balbi M, Ghersetti M, Grazioli S, Perciaccante A, et al. (2009) Antidiabetic therapy and increased risk of hepatocellular carcinoma in chronic liver disease. World J Gastroenterol 15: 2506-2511.

8. Neuschwander-Tetri BA, Clark JM, Bass NM, Van Natta ML, Unalp-Arida A et al. (2010) Clinical, laboratory and histological associations in adults with nonalcoholic fatty liver disease. Hepatology 52: 913-924.

9. American Diabetes Association (2013) Diagnosis and classification of diabetes mellitus. Diabetes Care 36 Suppl 1: S67-74.

10. Chobanian AV, Bakris GL, Black HR, Cushman WC, Green LA, et al. (2003) Seventh report of the Joint National Committee on Prevention, Detection, Evaluation, and Treatment of High Blood Pressure. Hypertension 42: 12061252

11. Kleiner DE, Brunt EM, Van Natta M, Behling C, Contos MJ, et al. (2005) Design and validation of a histological scoring system for nonalcoholic fatty liver disease. Hepatology 41: 1313-1321.

12. Angulo P, Hui JM, Marchesini G, Bugianesi E, George J, et al. (2007) The NAFLD fibrosis score: a noninvasive system that identifies liver fibrosis in patients with NAFLD. Hepatology 45: 846-854

13. Harrison SA, Oliver D, Arnold HL, Gogia S, Neuschwander-Tetri BA (2008) Development and validation of a simple NAFLD clinical scoring system for identifying patients without advanced disease. Gut 57:1441-1447.

14. McPherson S, Stewart SF, Henderson E, Burt AD, Day CP (2010) Simple noninvasive fibrosis scoring systems can reliably exclude advanced fibrosis in patients with non-alcoholic fatty liver disease. Gut 59:1265-1269.

15. Loomba R, Abraham M, Unalp A, Wilson L, Lavine J, et al. (2012) Association between diabetes, family history of diabetes, and risk of nonalcoholic steatohepatitis and fibrosis. Hepatology 56: 943-951.

16. Svegliati-Baroni G, Ridolfi F, Di Sario A, Casini A, Marucci L, et al. (1999) Insulin and insulin-like growth factor-1 stimulate proliferation and type I collagen accumulation by human hepatic stellate cells: differential effects on signal transduction pathways. Hepatology 29:1743-1751.

17. Paradis V, Perlemuter G, Bonvoust F, Dargere D, Parfait B, et al. (2001) High glucose and hyperinsulinemia stimulate connective tissue growth factor expression: a potential mechanism involved in progression to fibrosis in nonalcoholic steatohepatitis. Hepatology 34:738-744

18. Williams EJ, Gaça MD, Brigstock DR, Arthur MJ, Benyon RC (2000) Increased expression of connective tissue growth factor in fibrotic human liver and in activated hepatic stellate cells. J Hepatol 32: 754-761.

19. Gressner OA, Gressner AM (2008) Connective tissue growth factor: a fibrogenic master switch in fibrotic liver diseases. Liver Int 28: 1065-1079.

20. Donadon V, Balbi M, Mas MD, Casarin P, Zanette G (2010) Metformin and reduced risk of hepatocellular carcinoma in diabetic patients with chronic liver disease. Liver Int 30: 750-758.

21. Bowker SL, Majumdar SR, Veugelers P, Johnson JA (2006) Increased cancerrelated mortality for patients with type 2 diabetes who use sulfonylureas or insulin. Diabetes Care 29: 254-258.

22. Chang $\mathrm{CH}$, Lin JW, Wu LC, Lai MS, Chuang LM, et al. (2012) Association of thiazolidinediones with liver cancer and colorectal cancer in type 2 diabetes mellitus. Hepatology 55: 1462-1472

23. Singh S, Singh PP, Singh AG, Murad MH, Sanchez W (2013) Anti-diabetic medications and the risk of hepatocellular cancer: a systematic review and meta-analysis. Am J Gastroenterol 108: 881-891.

24. McGlynn KA, London WT (2005) Epidemiology and natural history of hepatocellular carcinoma. Best Pract Res Clin Gastroenterol 19: 3-23.

25. Cusi K (2012) Role of obesity and lipotoxicity in the development of nonalcoholic steatohepatitis: pathophysiology and clinical implications. Gastroenterology 142: 711-725.

26. Targher G, Day CP, Bonora E (2010) Risk of cardiovascular disease in patients with nonalcoholic fatty liver disease. N Engl J Med 363: 1341-1350.

27. Farrell G (2014) Should we lower lipids in nonalcoholic fatty liver disease? Clin Gastroenterol Hepatol 12: 152-155.

28. Athyros VG, Tziomalos K, Gossios TD, Griva T, Anagnostis P, et al. (2010) Safety and efficacy of long-term statin treatment for cardiovascular events in patients with coronary heart disease and abnormal liver tests in the Greek Atorvastatin and Coronary Heart Disease Evaluation (GREACE) Study: a posthoc analysis. Lancet 376:1916-1922.

29. Van Rooyen DM, Gan LT, Yeh MM, Haigh WG, Larter CZ, et al. (2013) Pharmacological cholesterol lowering reverses fibrotic NASH in obese, diabetic mice with metabolic syndrome. J Hepatol 59:144-152.

30. Ekstedt M, Franzén LE, Mathiesen UL, Holmqvist M, Bodemar G, et al. (2007) Statins in non-alcoholic fatty liver disease and chronically elevated liver enzymes: a histopathological follow-up study. J Hepatol 47: 135-141. 